\title{
Multi-Scale 3-D Free-Form Surface Smoothing
}

\author{
F. Mokhtarian, N. Khalili and P. Yuen \\ Centre for Vision Speech and Signal Processing \\ Dept. of Electronic and Electrical Engineering \\ University of Surrey, Guildford, Surrey GU2 5XH, UK \\ F.Mokhtariandee.surrey.ac.uk
}

\begin{abstract}
A novel technique for multi-scale smoothing of a free-form 3-D surface is presented. Complete triangulated models of 3-D objects are constructed (through fusion of range images) and are then described at multiple scales. This is achieved by convolving local parametrizations of the surface with 2-D Gaussian filters iteratively. Our method for local parametrization makes use of semigeodesic or goedesic polar coordinates as a natural and efficient way of sampling the local surface shape. The smoothing eliminates surface noise and small surface detail gradually. Our technique for 3-D multi-scale surface smoothing is independent of the underlying triangulation. It is also argued that the proposed technique is preferrable to volumetric smoothing or level set methods since it is applicable to incomplete surface data which occurs during occlusion.
\end{abstract}

\section{Introduction}

This paper introduces a new technique for multi-scale shape description of free-form 3-D surfaces represented by polygonal or triangular meshes. Complete 3-D models of test objects have been used in our experiments. Such models can be constructed through automatic fusion of range images of the object obtained from different viewpoints [4].

Multi-scale descriptions have become very common in computer vision since they offer added robustness with respect to noise and object detail as well as provide for more efficient processing. The multi-scale technique proposed here can be considered a generalization of earlier multi-scale representation theories proposed for 2-D contours $[8,9]$ and space curves [7]. However, the theoretical issues are significantly more challenging when working on free-form 3D surfaces.

In our approach, diffusion of the surface is achieved through convolutions of local parametrizations of the surface with a 2-D Gaussian filter. Semigeodesic coordinates [2] are utilized as a natural and efficient way of locally parametrizing surface shape. The most important advantage of our method is that unlike other diffusion techniques such as volumetric diffusion [6,5] or level set methods [12], it has local support and is therefore applicable to partial data corresponding to surface-segments. This property makes it suitable for object recognition applications in presence of occlusions. 
The organization of this paper is as follows. Section 2 gives a brief overview of previous work on 3-D object representations including the disadvantage(s) of each method. Section 3 describes the relevant theory from differential geometry and explains how a multi-scale shape description can be computed for a free-form 3-D surface. Both semigeodesic and geodesic polar coordinates are covered. Section 4 covers implementation issues encountered when adapting semigeodesic coordinates and geodesic polar coordinates to 3-D triangular meshes. Section 5 presents diffusion results and discussion. Section 6 contains the concluding remarks.

\section{Literature Survey}

Polyhedral approximations [1] fit a polyhedral object with vertices and relatively large flat faces to a 3-D object. Their disadvantage is that the choice of vertices can be quite arbitrary which renders the representation not robust. Smooth 3-D splines [14] can also be fitted to 3-D objects. Their shortcomings are that the choice of knot points is again arbitrary and that the spline parameters are not invariant. Generalized cones or cylinders [13] as well as geons [10] approximate a 3-D object using globally parametrized mathematical models, but they are not applicable to detailed free-form objects. Multi-view representations [11] are based on a large number of views of a 3-D object obtained from different viewpoints, but difficulties can arise when a non-standard view is encountered. In volumetric diffusion [5] or level set methods [12], an object is treated as a filled area or volume. The object is then blurred by subjecting it to the diffusion equation. The boundary of each blurred object can then be defined by applying the Laplacian operator to the smoothed area or volume. The major shortcoming of these approaches is lack of local support. In other words, the entire object data must be available. This problem makes them unsuitable for object recognition in presence of occlusion. A form of 3-D surface smoothing has been carried out in $[15,16]$ but this method has drawbacks since it is based on weighted averaging using neighboring vertices and is therefore dependent on the underlying triangulation.

\section{Semigeodesic and Geodesic Polar Parametrization on a 3-D Surface}

A crucial property of 2-D contours and space curves (or 3-D contours) is that they can be parametrized globally using the arclength parameter. However, free-form 3-D surfaces are more complex. As a result, no global coordinate system exists on a free-form 3-D surface which could yield a natural parametrization of that surface. Indeed, studies of local properties of 3-D surfaces are carried out in differential geometry using local coordinate systems called curvilinear coordinates or Gaussian coordinates [2]. Each system of curvilinear coordinates is introduced on a patch of a regular surface referred to as a simple sheet. A simple sheet of a surface is obtained from a rectangle by stretching, squeezing, and bending but without tearing or gluing together. Given a parametric representation $\mathbf{r}$ $=\mathbf{r}(u, v)$ on a local patch, the values of the parameters $u$ and $v$ determine the position of each point on that patch. 


\subsection{Geodesic Lines}

Before the semigeodesic and geodesic polar coordinates can be described, it is necessary to define geodesic lines on a regular 3-D surface. The following definitions are useful $[2,3]$ :

Definition. A geodesic line or a geodesic of a surface is a curve whose geodesic curvature is zero at every point. Geodesic curvature is the magnitude of the vector of geodesic curvature.

Definition. The vector of geodesic curvature of a curve $C$ lying on a surface $\mathcal{S}$ at a point $P$ on $C$ is obtained by projecting the curvature vector of $C$ at $P$ on the tangent plane to $\mathcal{S}$ at $P$.

Definition. The curvature vector of a curve $C$ at point $P$ is of the same direction as the principal normal vector at $P$ and of length equal to the curvature of the curve at $P$.

Definition. The principal normal vector of a curve $C$ at point $P$ is perpendicular to $C$ at $P$ and lies in the osculating plane at $P$. The plane with the highest possible order of contact with the curve $C$ at point $P$ is called the osculating plane at $P$.

The following crucial property of geodesic lines is actually utilized to construct geodesics on 3-D triangular meshes:

Minimal property of geodesics: An arc of a geodesic line $C$ passing through a point $P$ and lying entirely in a sufficiently small neighborhood of a point $P$ of a surface $\mathcal{S}$ of class $C_{2}$ is the shortest join of $P$ with any other point of $C$ by a curve lying in the neighborhood.

\subsection{Semigeodesic Coordinates}

Semigeodesic coordinates can be constructed in the following way at a point $P$ on a surface $\mathcal{S}$ of class $C_{2}$ :

- Choose a geodesic line $C$ through point $P$ in an arbitrary direction.

- Denote by $v$ the arclength parameter on $C$, such that $P$ corresponds to the value $v=0$.

- Take further through every point of $C$ the geodesic line $L$ perpendicular to $C$ at the corresponding point.

- Denote by $u$ the arclength parameter on $L$.

The two parameters $u$ and $v$ determine the position of each point in the domain swept out by these geodesic lines. It can be shown that in a sufficiently small neighborhood of the point $P$, semigeodesic coordinates can always serve as curvilinear coordinates in a regular parametric representation of $\mathcal{S}$ [2]. The orthogonal cartesian coordinates in the plane are a special case of semigeodesic coordinates on a flat surface.

\subsection{Geodesic Polar Coordinates}

Geodesic Polar coordinates can be constructed at a point $P$ on a surface $\mathcal{S}$ of class $C_{2}$ in the following way:

- Choose an arbitrary direction $\mathbf{w}$ on $\mathcal{S}$ at point $P$. 
- Take all geodesic lines emanating from point $P$.

- Denote by $v$ the arclength parameter on each geodesic in previous step.

- Denote by $u$ the angle between $\mathbf{w}$ and the tangent vector of each geodesic in step 2 at point $P$.

Again the two parameters $u$ and $v$ determine the position of each point in the domain swept out by these geodesic lines. It can be shown that in a sufficiently small neighborhood of point $P$ (with $P$ itself deleted), geodesic polar coordinates can always serve as curvilinear coordinates. Point $P$ is a singular point of this parametrization since its coordinates are not uniquely defined. The polar coordinates in the plane are a special case of geodesic polar coordinates on a flat surface.

\subsection{Gaussian Smoothing of a 3-D surface}

The procedures outlined above can be followed to construct semigeodesic coordinates or geodesic polar coordinates at every point of a 3-D surface $\mathcal{S}$. In case of semigeodesic coordinates, local parametrization yields at each point $P$ :

$$
\mathbf{r}(u, v)=(x(u, v), y(u, v), z(u, v)) .
$$

The new location of point $P$ is given by:

$$
\mathbf{R}(u, v, \sigma)=(\mathcal{X}(u, v, \sigma), \mathcal{Y}(u, v, \sigma), \mathcal{Z}(u, v, \sigma))
$$

where

$$
\begin{gathered}
\mathcal{X}(u, v, \sigma)=x(u, v) \otimes G(u, v, \sigma) \\
\mathcal{Y}(u, v, \sigma)=y(u, v) \otimes G(u, v, \sigma) \\
\mathcal{Z}(u, v, \sigma)=z(u, v) \otimes G(u, v, \sigma) \\
G(u, v, \sigma)=\frac{1}{2 \pi \sigma^{2}} e^{-\frac{\left(u^{2}+v^{2}\right)}{2 \sigma^{2}}}
\end{gathered}
$$

and $\otimes$ denotes convolution. In case of geodesic polar coordinates, the Gaussian function becomes one-dimensional. As a result, each of the 2-D convolutions above can be expressed as a series of 1-D convolutions.

In both cases, this process is repeated at each point of $\mathcal{S}$ and the new point positions after filtering define the smoothed surface. Since the coordinates constructed are valid locally, the Gaussian filters always have $\sigma=1$.

\subsection{Multi-Scale Description of a 3-D surface via diffusion}

In order to achieve multi-scale descriptions of a 3-D surface $\mathcal{S}$, it is smoothed according to the process described in section 3.4. The smoothed surface is then considered as the input to the next stage of smoothing. This procedure is then iterated many times to obtain multi-scale descriptions of $\mathcal{S}$. This process is equivalent to diffusion smoothing

$$
\frac{\partial \mathcal{S}}{\partial t}=H \mathbf{n}
$$


since the Gaussian function satisfies the heat equation. In the equation above, $t$ is time, $H$ is mean curvature, and $\mathbf{n}$ is the surface normal vector. $t$ can be regarded as the number of iterations.

\section{Implementation on a 3-D Triangular Mesh}

The theory explained in the previous section must be adapted to a 3-D triangular mesh. Both semigeodesic and geodesic polar coordinates involve construction of geodesic lines. Clearly the segment of a geodesic that lies on any given triangle is a straight line. Two situations must be considered:

- Extension of a geodesic when it intersects a triangle edge

- Extension of a geodesic when it intersects a triangle vertex

Theorem 1 addresses the first situation.

Theorem 1. Suppose a geodesic intersects an edge $e$ shared by triangles $T_{1}$ and $T_{2}$. The extension of this geodesic beyond $e$ is obtained by rotating $T_{2}$ about $e$ so that it becomes co-planar with $T_{1}$, extending the geodesic in a straight line on $T_{2}$, and rotating $T_{2}$ about $e$ back to its original position.

Proof: Assume by contradiction that the procedure above does not construct a geodesic. Let $g_{1}$ be the segment of the geodesic on $T_{1}$ and let $g_{2}$ be the segment of the geodesic on $T_{2}$. Rotate $T_{2}$ about $e$ so that it becomes coplanar with $T_{1}$. By assumption, $g_{1}$ and $g_{2}$ will not be co-linear. Hence, for a point $P_{1}$ on $g_{1}$ and a point $P_{2}$ on $g_{2}$, there will be a shorter path from $P_{1}$ to $P_{2}$. This is the straight line joining $P_{1}$ to $P_{2}$. Now rotate $T_{2}$ back to its original position. The length of the path just constructed remains the same, so it will still be shorter than the geodesic from $P_{1}$ to $P_{2}$. A contradiction has been reached. Therefore the procedure described correctly constructs a geodesic.

Note that the construction above extends to several triangles as long as they remain in a local neighborhood. Theorem 2 addresses the second case.

Theorem 2. Suppose a geodesic arrives at a vertex $V$ of the mesh. Define the normal vector $\mathbf{n}$ at $V$ as the average of the surface normals of all the triangles incident on $V$ weighted by the incident angle. Let $Q$ be the plane formed by the geodesic incident on $V$ and $\mathbf{n}$. The extension of this geodesic beyond $V$ is found by intersecting $Q$ with the mesh.

Proof: The curvature vector $k$ of the path obtained by the procedure above lies in $Q$. $k$ is also perpendicular to tangent plane $T$ (which is defined as perpendicular to $\mathbf{n}$ at $V$ ). The vector of geodesic curvature of the path is obtained by projecting $k$ on the tangent plane. It follows that geodesic curvature of the path is zero. Hence the path is a geodesic line.

\subsection{Implementation of semigeodesic coordinates}

Semigeodesic coordinates are constructed at each vertex of the mesh which becomes the local origin. The following procedure is employed:

- Construct a geodesic from the origin in an arbitrary direction such as the direction of one of the incident edges. 


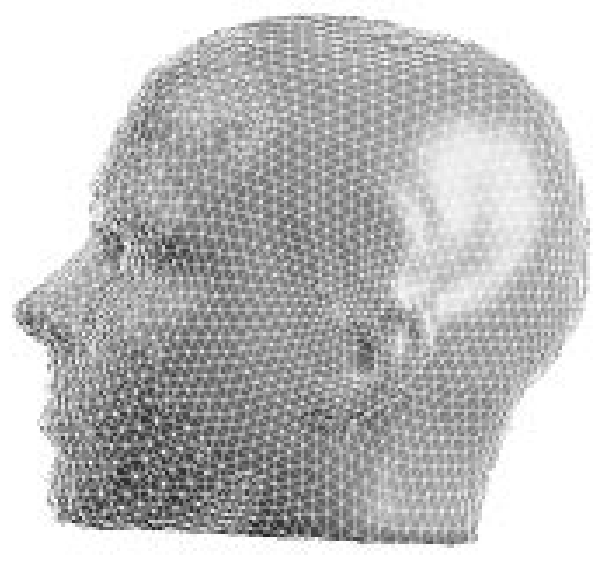

(a) A 3-D triangular mesh

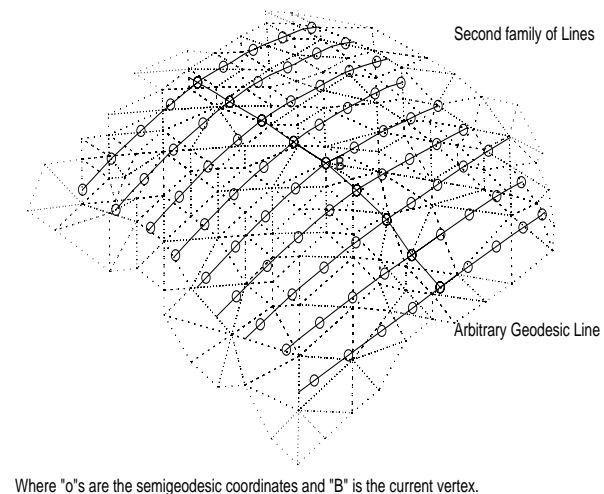

(b) Semigeodesic coordinates

Figure 1: A triangular mesh and semigeodesic coordinates in a local area

- Construct the other half of that geodesic by extending it through the origin in the reverse direction using the procedure outlined in theorem 2 .

- Parametrize that geodesic by the arclength parameter at regular intervals to obtain a sequence of sample points.

- At each sample point on the first geodesic, construct a perpendicular geodesic and extend it in both directions.

- Parametrize each of the geodesics constructed in the previous step by the arclength parameter at regular intervals.

Figure 1 shows a triangular mesh and an example of semigeodesic coordinates.

\subsection{Implementation of geodesic polar coordinates}

Geodesic polar coordinates are also constructed at each vertex of the mesh which again becomes the local origin. The following procedure is used:

- Construct a geodesic from the origin in an arbitrary direction such as the direction of one of the incident edges.

- Let $N$ be the normal plane at the origin defined by the geodesic constructed in the previous step and the normal vector $\mathbf{n}$ (defined in theorem 2).

- Rotate $N$ about $\mathbf{n}$ by angle $\alpha$ and intersect it with the mesh to obtain the next geodesic emanating from the origin. 
- Repeat the previous step until $N$ is back in its original position.

- Parametrize each of the constructed geodesics by the arclength parameter at regular intervals to obtain a sequence of sample points on each geodesic.

\section{Results and Discussion}

The smoothing routines were implemented entirely in $\mathrm{C}++$. The first test object was a cube with 98 vertices and 192 triangles. The smoothing results using semigeodesic coordinates (with filter size equal to 9) are shown in Figure 2 (top row). The original cube is changed to a sphere after five iterations. The experiment was also repeated using geodesic polar coordinates (with 9 polar lines), and the smoothing results are shown in Figure 2 (bottom row). These results indicate that smoothing using semigeodesic coordinates and geodesic polar coordinates produce similar results. The second test object was a foot with 2898 triangles and 1451 vertices. The smoothing results are shown in Figure 3. The third test object was a chair with 3788 triangles and 1894 vertices as shown in Figure 4. Figure 5 shows the fourth test object which was a telephone handset with 11124 tringles and 5564 vertices. These examples show that our technique is effective in eliminating surface noise as well as removing surface detail. The result is gradual simplification of object shape. Animation of surface diffusion can be observed at the web site:

http://www.ee.surrey.ac.uk/Research/VSSP/demos/css3d/index.html

\section{Conclusions}

A novel technique for multi-scale smoothing of a free-form triangulated 3-D surface was presented. The method was independent of the underlying triangulation. This was achieved by convolving local parametrizations of the surface with 2-D Gaussian filters iteratively. Our method for local parametrization made use of semigeodesic and geodesic polar coordinates as natural and efficient ways of sampling the local surface shape. Smoothing techniques using semigeodesic coordinates and geodesic polar coordinates produce similar results. The smoothing eliminated the surface noise and small surface detail gradually, and resulted in gradual simplification of object shape. Our approach is preferrable to volumetric smoothing or level set methods since it is applicable to incomplete surface data which occurs during occlusion.

\section{References}

[1] O D Faugeras and M Hebert. The representation, recognition, and locating of 3-d objects. International Journal of Robotics Research, 5(3):27-52, 1986.

[2] A Goetz. Introduction to Differential Geometry. Addison-Wesley, 1970.

[3] S Heitzz. Coordinates in Geodesy. Addison-Wesley, Berlin, 1985.

[4] A Hilton, A J Stoddart, J Illingworth, and T Windeatt. Reliable surface reconstruction from multiple range images. In Proc ECCV, Cambridge, UK, 1996.

[5] J J Koenderink. Solid Shape. MIT Press, Cambridge, MA, 1990. 


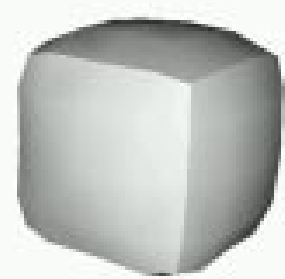

(a) 1 iteration

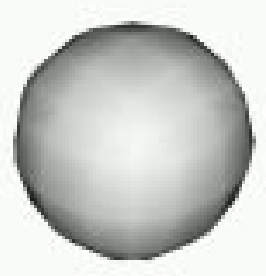

(b) 5 iterations

Semigeodesic coordinates

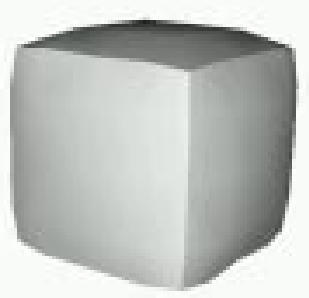

(c) 1 iteration

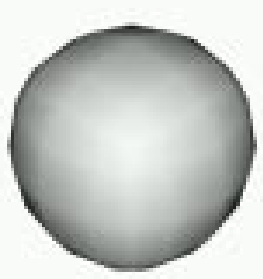

(d) 5 iterations

Geodesic Polar coordinates

Figure 2: Smoothing of the Cube

[6] J J Koenderink and A J vanDoorn. Dynamic shape. Biological Cybernetics, 53:383-396, 1986.

[7] F Mokhtarian. A theory of multi-scale, torsion-based shape representation for space curves. Computer Vision and Image Understanding, 68(1):1-17, 1997.

[8] F Mokhtarian and A K Mackworth. newblock Scale-based description and recognition of planar curves and two-dimensional shapes. IEEE Trans Pattern Analysis and Machine Intelligence, 8(1):34-43, 1986.

[9] F Mokhtarian and A K Mackworth. A theory of multi-scale, curvature-based shape representation for planar curves. IEEE Trans PAMI, 14(8):789-805, 1992.

[10] M Pilu and R Fisher. Recognition of geons by parametric deformable contour models. In Proc ECCV, pages 71-82, Cambridge, UK, 1996.

[11] M Seibert and A M Waxman. Adaptive 3-d object recognition from multiple views. IEEE Trans Pattern Analysis and Machine Intelligence, 14:107-124, 1992. 


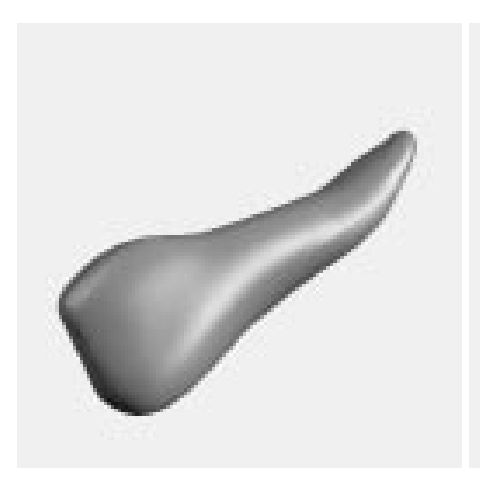

(a) Original

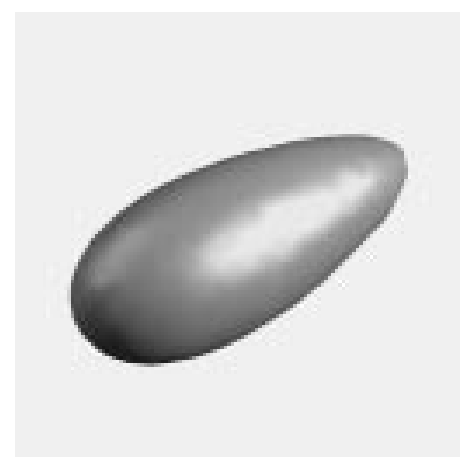

(c) 40 iterations

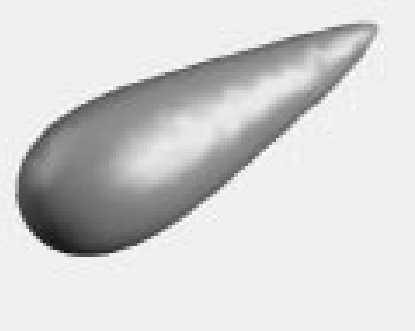

(b) 20 iterations

Figure 3: Diffusion of the Foot

[12] J A Sethian. Level Set Methods. Cambridge University Press, 1996.

[13] B I Soroka and R K Bajcsy. Generalized cylinders from serial sections. In Proc IJCPR, 1976.

[14] A J Stoddart and M Baker. Reconstruction of smooth surfaces with arbitrary topology and aptive splines. In Proc ECCV, 1998.

[15] G Taubin. Curve and surface smoothing without shrinkage. In Proc ICCV, pages 852-857, 1995.

[16] G Taubin. Optimal surface smoothing as filter design. In Proc ECCV, 1996. 


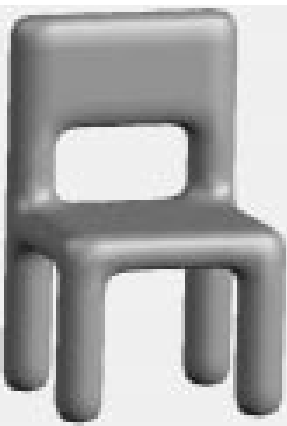

(a) Original

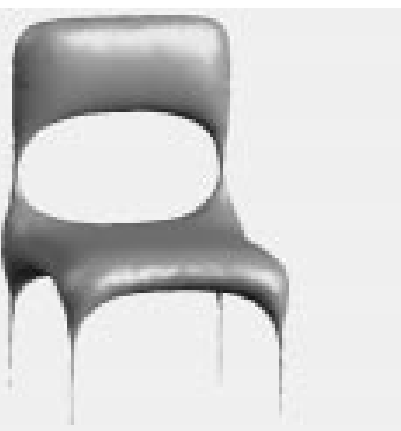

(b) 4 iterations

Figure 4: Smoothing of the Chair

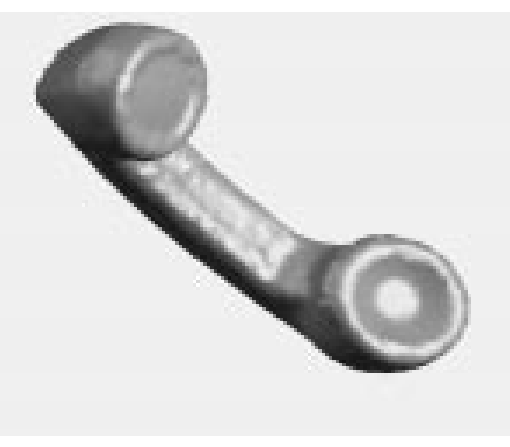

(a) Original

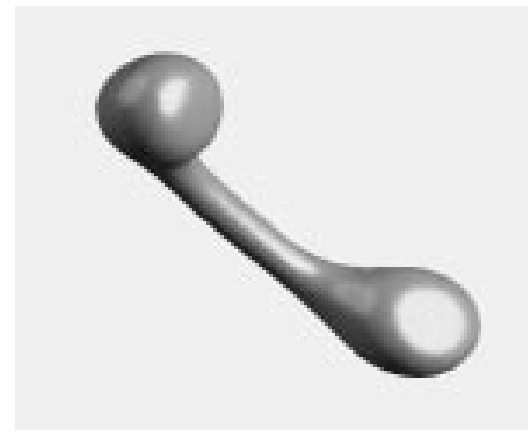

(c) 18 iterations

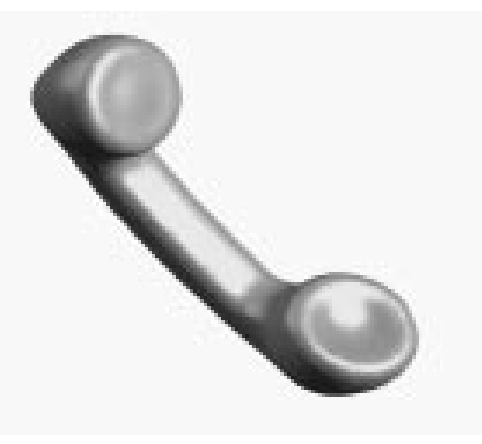

(b) 3 iterations

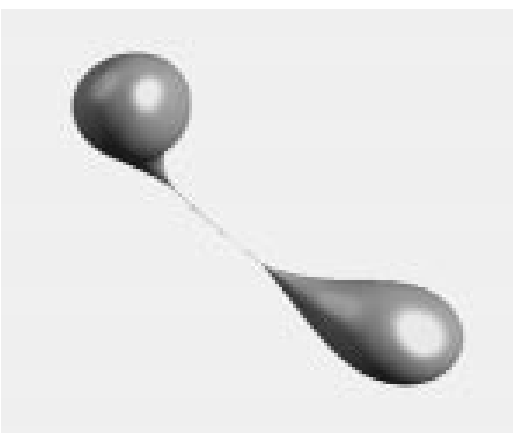

(d) 31 iterations

Figure 5: Diffusion of the telephone handset 